\title{
Time-Frequency Characterization of Cerebral Hemodynamics of Migraine Sufferers as Assessed by NIRS Signals
}

\author{
Filippo Molinari, ${ }^{1}$ Samanta Rosati, ${ }^{1}$ William Liboni, ${ }^{2}$ Emanuela Negri, ${ }^{2}$ Ornella Mana, ${ }^{2}$ \\ Gianni Allais, ${ }^{3}$ and Chiara Benedetto ${ }^{3}$ \\ ${ }^{1}$ Biolab, Department of Electronics, Polytechnic of Turin, 10129 Torino, Italy \\ ${ }^{2}$ Department of Neuroscience, Gradenigo Hospital, 10153 Turin, Italy \\ ${ }^{3}$ Women's Headache Center, Department of Gynecology and Obstetrics, University of Torino, 10126 Torino, Italy
}

Correspondence should be addressed to Filippo Molinari, filippo.molinari@polito.it

Received 31 December 2009; Accepted 24 June 2010

Academic Editor: L. F. Chaparro

Copyright (C) 2010 Filippo Molinari et al. This is an open access article distributed under the Creative Commons Attribution License, which permits unrestricted use, distribution, and reproduction in any medium, provided the original work is properly cited.

Near-infrared spectroscopy (NIRS) is a noninvasive system for the real-time monitoring of the concentration of oxygenated $\left(\mathrm{O}_{2} \mathrm{Hb}\right)$ and reduced $(\mathrm{HHb})$ hemoglobin in the brain cortex. $\mathrm{O}_{2} \mathrm{Hb}$ and $\mathrm{HHb}$ concentrations vary in response to cerebral autoregulation. Sixty-eight women (14 migraineurs without aura, 49 migraineurs with aura, and 5 controls) performed breathholding and hyperventilation during NIRS recordings. Signals were processed using the Choi-Williams time-frequency transform in order to measure the power variation of the very-low frequencies (VLF: $20-40 \mathrm{mHz}$ ) and of the low frequencies (LF: $40-140 \mathrm{mHz}$ ). Results showed that migraineurs without aura present different LF and VLF power levels than controls and migraineurs with aura. The accurate power measurement of the time-frequency analysis allowed for the discrimination of the subjects' hemodynamic patterns. The time-frequency analysis of NIRS signals can be used in clinical practice to assess cerebral hemodynamics.

\section{Introduction}

Autoregulation of blood flow denotes the intrinsic ability of an organ or a vascular bed to maintain a constant perfusion in presence of blood pressure changes and metabolic demand $[1,2]$. In particular, the mechanism of cerebral autoregulation represents the tendency of cerebral blood flow to remain relatively constant, despite changes in mean arterial blood pressure and neuronal activity [3]. This mechanism is particularly important for the human brain, since it represents a protection condition against sudden and abrupt arterial blood pressure changes and intracranial pressure disturbances. The autoregulatory mechanism acts by tuning the vasodilation and vasoconstriction of the cerebral microvessels [4]. This activity, usually called vasomotor reactivity, determines the blood volume supplied to the brain tissue, thus fixing the total available oxygen. In some pathologic conditions, autoregulation may be impaired or even lost $[1,5,6]$.
The assessment of autoregulation is usually made by means of active stimuli [4]. Breath-holding (BH) is effective in triggering autoregulation, since the increase of the carbon dioxide in the blood determines a vasodilation of the cerebral vessels. Conversely, hyperventilation (HYP) triggers vasoconstriction, since the increase of oxygen in the blood determines a reduction of the cerebral blood flow. The quantification of autoregulation can be done either by measuring the changes in the cerebral blood flow velocity in the brain arteries (by means of transcranial Doppler sonography $[4,7,8]$ ) or by measuring the concentration of oxygen and carbon dioxide in brain tissue (by means of nearinfrared spectroscopy [9-13]). Specifically, near-infrared spectroscopy (NIRS) systems allow for the noninvasive realtime monitoring of the concentration of oxygenated and reduced hemoglobin in brain cortex. The subject's autoregulatory capability is assessed by measuring the changes in the oxygen content, carbon dioxide content, and cerebral blood flow velocity during an active stimulus like $\mathrm{BH}$ or HYP 
[14-16]. All the above-mentioned indicators are derived by the signals' time course.

Several studies documented the altered autoregulation, and consequent altered vasomotor reactivity, in migraine sufferers [17-19]. Migraine, in fact, is now considered essentially as a neurovascular pathology [19]. Results are not consistent in literature, since the testing procedures may vary from group to group. In previous studies, we documented a limited vasodilation capability in migraine sufferers [12], but Vernieri et al. recently found an increase in the vascular response of migraineurs [20], possibly mediated by a dysfunction in the autonomic control. Such experiments, conducted on groups of patients, document the limited reliability of time-derived parameters when used to assess autoregulation.

In 2000, Obrig et al. [21] studied the spontaneous low frequency oscillations of cerebral hemodynamics and metabolism in adult human head by using NIRS. Though conducted on healthy volunteers, this study introduced the possibility of frequency-derived parameters used to assess cerebral autoregulation. It is known that cerebral hemodynamic signals have a power spectrum essentially consisting of two different bands [22].

(1) A very low frequency band (VLF - also called Bwaves) that reflects the long-term autoregulation. At brain level, VLFs are thought to be generated by brain stem nuclei, which modulate the lumen of the small intracerebral vessels. In humans, the VLF is usually comprised between 20 and $40 \mathrm{mHz}$.

(2) A low frequency band (LF - also called M-waves) that is common to most mammalians. Such waves reflect the systemic oscillations of the arterial blood pressure and are modulated by the sympathetic system activity. LFs spans from about 40 to $140 \mathrm{mHz}$.

The above-described frequency bands can be observed on most of in vivo instrumental recordings, comprising transcranial Doppler, functional magnetic resonance, NIRS, laser-Doppler flowmetry, fluororeflectometry, and optical imaging [21]. Unfortunately, almost all the above-cited techniques provide nonstationary signals. An example of NIRS signals recorded during the $\mathrm{BH}$ (panel A) and HYP (panel B) of a healthy volunteer is shown in Figure 1. The nonstationarity affecting the signals is evident; therefore, a proper spectral analysis must be carried out using a joint time and frequency approach.

In this paper, we applied a time-frequency analysis procedure to NIRS signals recorded on a sample population of subjects affected by migraine with (MwA) and without (MwoA) aura. Aura is a specific disturbance associated with migraine that can cause visual, speech, or perceptional impairments. It has been proven that aura determines an alteration in the subjects' cerebral hemodynamics. Even if cerebral autoregulation impairment has been observed during MwA attacks, it is still unclear whether MwA sufferers present a normal autoregulation during attack-free periods [20]. The aim of our study was twofold: (i) first, to accurately measure the VLF and LF power changes in the NIRS signals

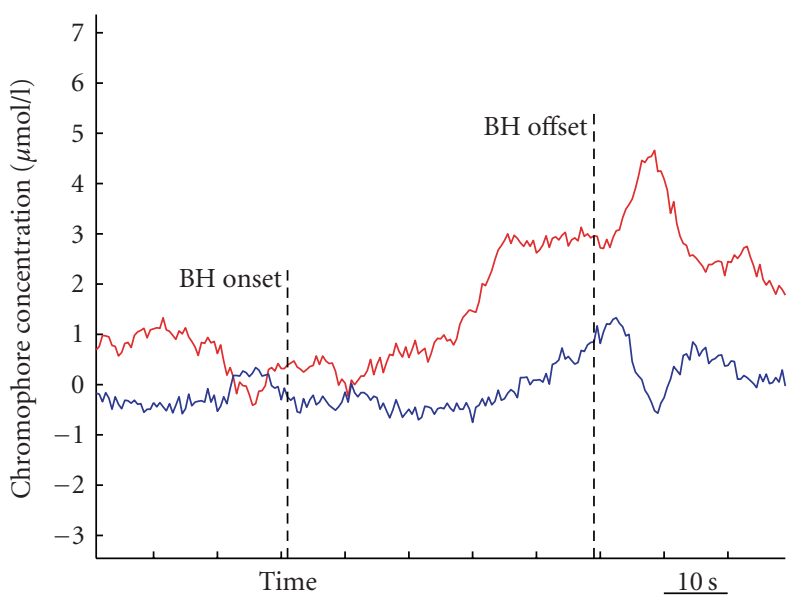

(a)

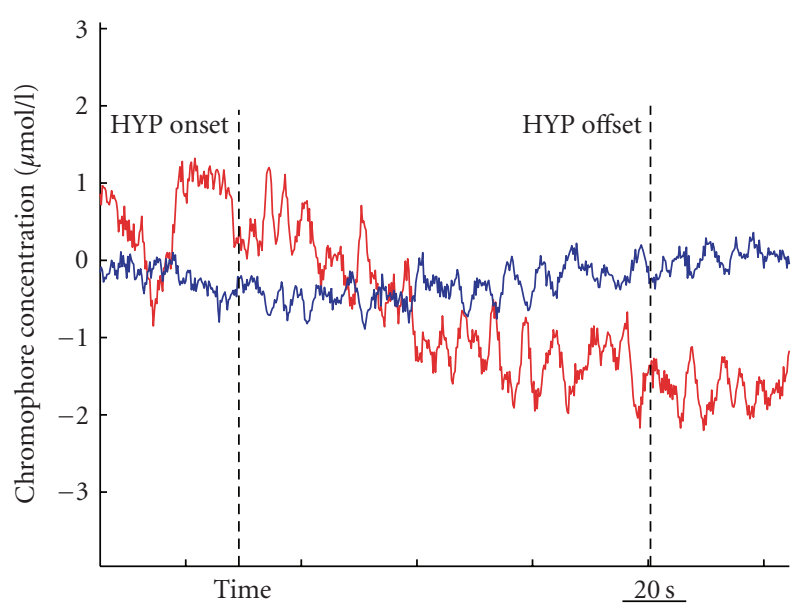

(b)

FIGURE 1: NIRS signals recorded on a healthy woman performing breath-holding (a) and hyperventilation (b). The red line represents the $\mathrm{O}_{2} \mathrm{Hb}$ concentration signal, the blue line the HHb. The black vertical dashed lines mark the onset and offset of the breath-holding (a) and hyperventilation (b). The graphs show that the NIRS signals become nonstationary as consequence of the active stimuli.

of migraineurs during active stimulations; and (ii) second, to document possible differences in the cerebral hemodynamics of MwA and MwoA sufferers.

The paper is organized as follows: in Section 2, the basics of NIRS devices and experimental procedures will be presented, along with the description of the time-frequency analysis procedure and the statistical tests. Section 3 will describe the results in terms of different hemodynamic patterns and spectral analyses, whereas Section 4 will discuss the results and the importance of a time-frequency analysis of NIRS signals in pathology. Section 5 will conclude the paper.

\section{Materials and Methods}

2.1. NIRS Recording and Experimental Protocol. NIRS is a spectroscopic technique that allows for the noninvasive and 

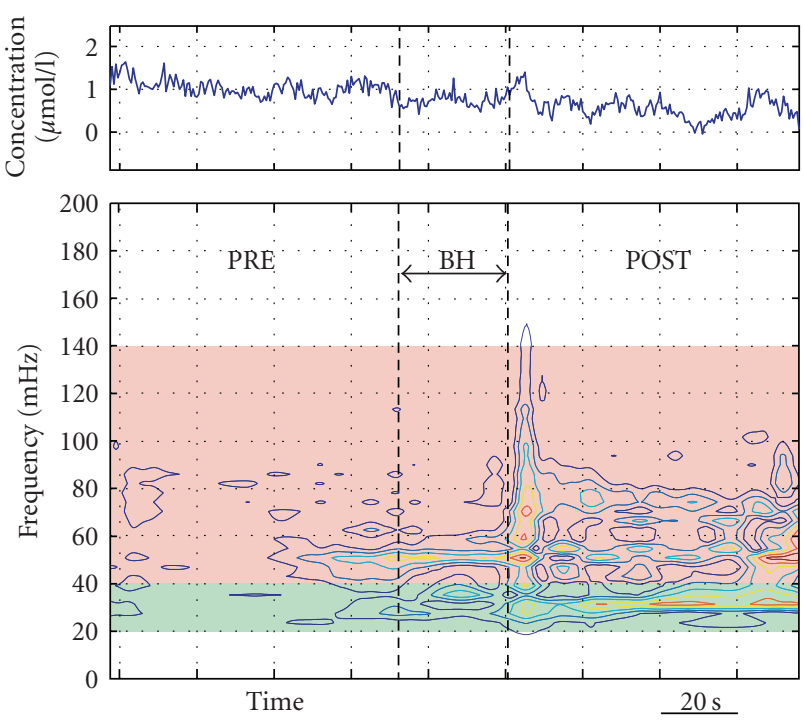

FIGURE 2: HHb concentration signal (upper panel) recorded on a healthy woman before (PRE), after (POST) and during breathholding $(\mathrm{BH})$. The vertical dashed lines mark the onset and offset of the $\mathrm{BH}$. the Lower panel shows the 6-levels contour plot of the Choi-Williams time-frequency distribution of the signal $(\sigma=0.05)$. The red rectangle indicates the LF band $(40-140 \mathrm{mHz})$, the green the VLF band $(20-40 \mathrm{mHz})$. In this subject, there is a neat increase of the VLF and LF power after $\mathrm{BH}$ (POST region).

real time monitoring of the concentration of oxygenated $\left(\mathrm{O}_{2} \mathrm{Hb}\right)$ and reduced $(\mathrm{HHb})$ hemoglobin in the human brain. Since the two types of hemoglobin have different absorption peaks, by using two light wavelengths, it is possible to monitor their concentration changes. A substance interacting with a particular wavelength is called "chromophore". Previous studies demonstrated that when monitoring human brain by using NIRS, the most important chromophores are $\mathrm{O}_{2} \mathrm{Hb}, \mathrm{HHb}$, and the cytochrome-coxidase (which is a neuronal metabolic marker). Other absorbers such as water, lipids, plasma, muscles, and bones can be neglected since their absorption peaks are far from the infrared region $[23,24]$. In this study, we will not consider cytochrome-c-oxidase data since they are more linked to a functional aspect of brain functioning rather than to hemodynamics.

In NIRS systems, a beam of light in the infrared band (wavelengths ranging from $650 \mathrm{~nm}$ to $870 \mathrm{~nm}$ are usually used) is injected into the skull by a photoemitter placed on the scalp. The light photons traveling into the skull are partly absorbed and partly scattered. A photodetector placed few centimeters far from the emitter acquires the photons emerging from the skull. The intensity of the measured light is indicative of the concentration of a given absorber. Unlike other spectroscopic systems, the NIRS devices usually adopt a scattering-based measurement and not a transmissionbased measurement (i.e., the photodetector is not placed controlateral to the source), therefore the traditional absorption equation cannot be used to measure the chromophore

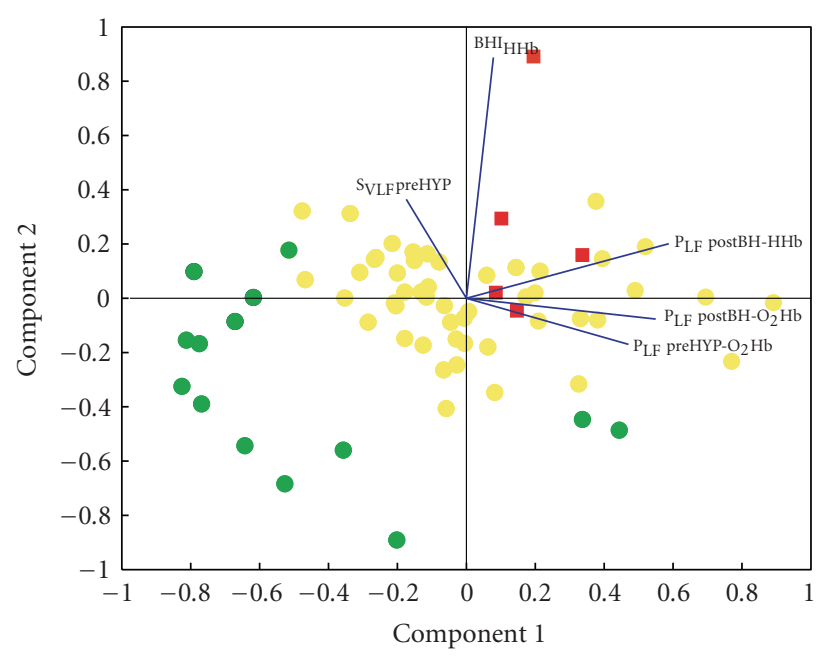

(a)

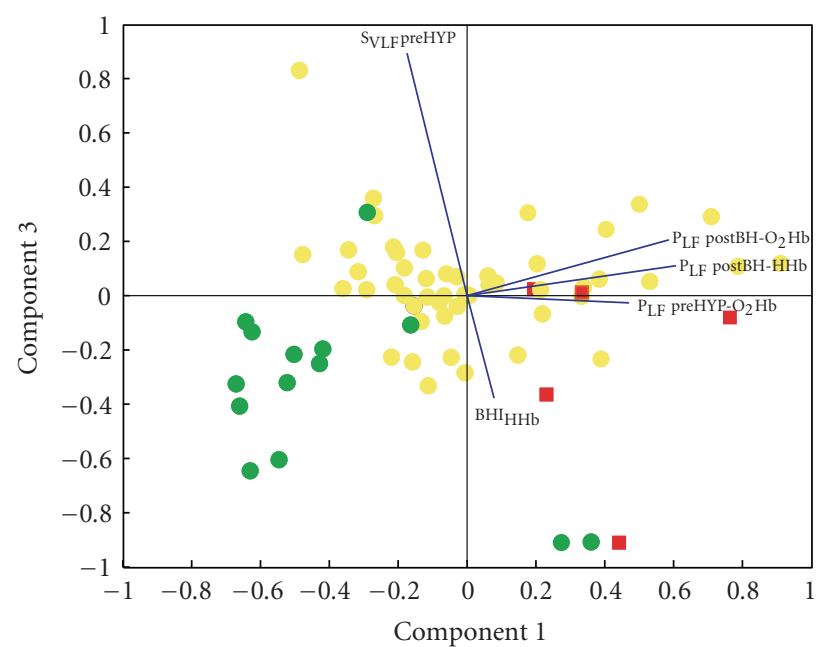

(b)

FIgure 3: Principal component representation of the subjects in the hyperplanes formed by (a) the 1st and 2nd components, and (b) the 1 st and 3rd components. Red squares indicate the healthy controls, green circles the migraine without aura patients, and the yellow circles the migraine with aura patients. The blue lines represent the loading/loading plot of the PCA: the lines indicate the direction of the original variables in the hyperplanes. The length of the blue lines projected onto the axis is proportional to the weight of the original variable for the specific component. Migraine without aura subjects (green circles) are clearly clustered far from the other subjects.

concentrations changes. The traditional absorption BeerLambert law, is redefined in the following modified way (modified Beer-Lambert law)

$$
\Delta A(\lambda)=L(\lambda) \ln (10) \sum_{i} \varepsilon_{i}(\lambda) \Delta c_{i}
$$

where

(i) $\Delta A(\lambda)$ is the attenuation change at the wavelength $\lambda$;

(ii) $L(\lambda)$ is the total pathlength $(\mathrm{mm})$ traveled by the photons at wavelength $\lambda$; 

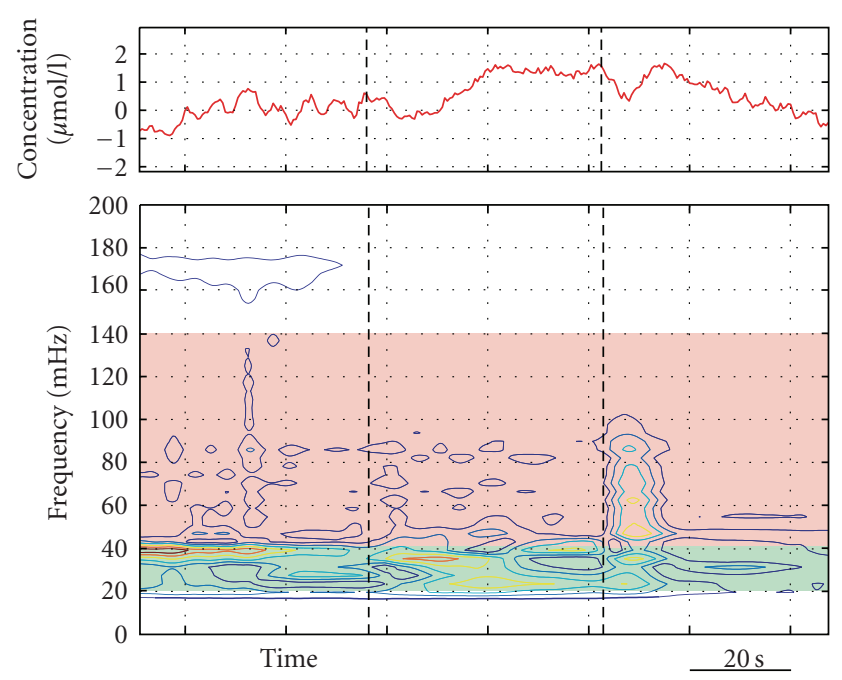

(a)
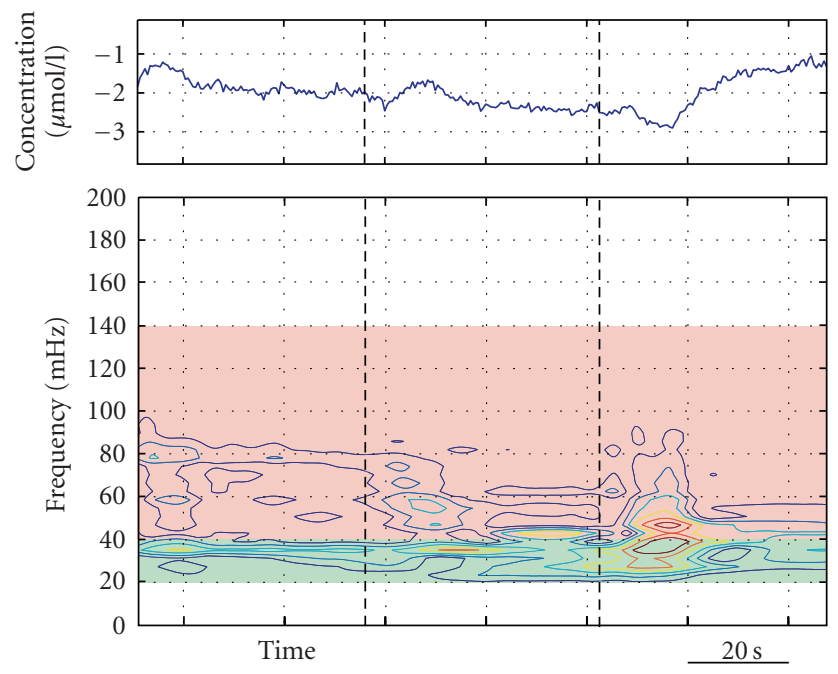

(b)

FIgURE 4: Time-frequency representations of $\mathrm{O}_{2} \mathrm{Hb}$ (a) and $\mathrm{HHb}$ (b) concentration signals during $\mathrm{BH}$. The graphs are relative to a subject suffering from migraine without aura. The vertical dashed lines mark the $\mathrm{BH}$ onset and offset. The red rectangle indicates the LF band $(40-140 \mathrm{mHz})$, the green the VLF band $(20-40 \mathrm{mHz})$. It is possible to notice that $\mathrm{BH}$ seems to decrease the spectral content of the signals in the LF band.

(iii) $\Delta c_{i}$ is the concentration change $\left(\mu \mathrm{mol} \cdot \mathrm{l}^{-1}\right)$ of the $i$ th chromophore at the wavelength $\lambda$;

(iv) $\varepsilon_{i}(\lambda)$ is the decadic extinction coefficient $\left(\mu \mathrm{mol}^{-1}\right.$. $\left.1 \cdot \mathrm{mm}^{-1}\right)$ of the $i$ th chromophore at the wavelength $\lambda$.

The attenuation is linearly dependent on the chromophores concentration changes; therefore, by measuring the light attenuation and solving the system in (1), it is possible to measure $\Delta c_{i}$. The total distance $L(\lambda)$ the photons travel into brain depends on the source-detector distance increased by a specific contribution given by scattering. This multiplier is called the differential pathlength factor.
Okada et al. proposed a differential pathlength factor value of 5.97 for infrared scattering in a model of adult human head [25].

We used a commercially available NIRS device (NIRO300, Hammamatsu Photonics, Australia) equipped by a 3-wavelengths source. The emitting probe of the NIRS equipment was placed on the left frontal side of the subjects, $2 \mathrm{~cm}$ beside the midline and about $3 \mathrm{~cm}$ above the supraorbital ridge. We chose this positioning in order to avoid the sinuses and to place the probes on a poorly perfused and very thin skin layer. The receiving sensor was fixed laterally to the emitter at a distance of about $5 \mathrm{~cm}$. To avoid bias from environmental light, a black cloth covered the NIRS probe. Chromophores concentration changes were acquired continuously at a sampling rate equal to $2 \mathrm{~Hz}$, discretized by a 16-bit A/D converter, lowpass filtered at $350 \mathrm{mHz}$ by means of an ARMA Chebychev filter with ripple in the stopband, and transferred to a laptop (by using a serial link) for further processing.

The recordings took place in a quiet and dimmed room with a constant temperature of $24-25^{\circ} \mathrm{C}$. The subjects were lying in supine position with eyes closed and breathing room air. All the subjects performed the following experimental protocol:

(1) 120 seconds of resting;

(2) a voluntary breath-holding followed by other 120 seconds of resting;

(3) a voluntary hyperventilation at the constant rate of about 20 respiratory acts per minute;

(4) a final resting period of 120 seconds.

The $\mathrm{BH}$ and HYP maneuvers were used to trigger cerebral autoregulation, since it is proven that $\mathrm{BH}$ induces vasodilation and HYP vasoconstriction $[5,11]$.

2.2. Time-Frequency Analysis of NIRS Signals. Figure 1 reports sample NIRS signals of a healthy volunteer performing $\mathrm{BH}$ (Figure 1(a)) and HYP (Figure 1(b)). The red line reports the $\mathrm{O}_{2} \mathrm{Hb}$ concentration variation during time, the blue the HHb. The vertical dashed lines mark the onset and offset of the BH (Figure 1(a)) and HYP (Figure 1(b)). The hemoglobin concentration significantly varies during time: in Figure 1(a), vasodilation corresponds to an increase in the $\mathrm{O}_{2} \mathrm{Hb}$ and a decrease in the $\mathrm{HHb}$ concentrations, whereas in Figure 1(b), vasoconstriction corresponds to a decrease in the $\mathrm{O}_{2} \mathrm{Hb}$ and an increase in the $\mathrm{HHb}$ concentrations. In Figure 1(b), the concentration signals are dominated by a harmonic trend that is synchronous with the forced respiratory rate.

The inner structure of the NIRS signals recorded during active maneuvers (BH and HYP) is clearly different from the one corresponding to the resting state. Therefore, these signals cannot be considered as stationary, not even in a wide-sense. We chose to process such signals using the time-frequency distributions belonging to the Cohen's class [26]. The definition of a generic bilinear time-frequency 

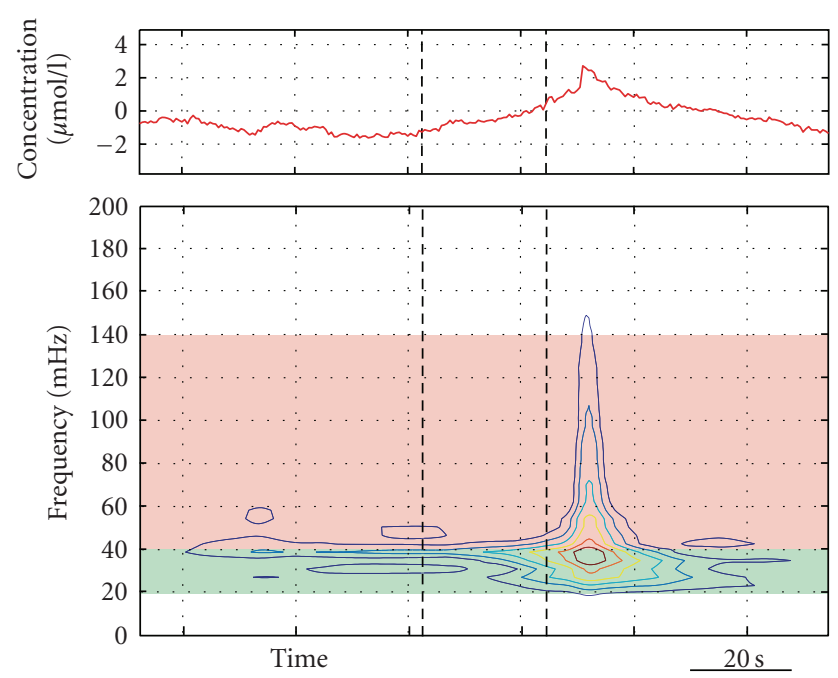

(a)
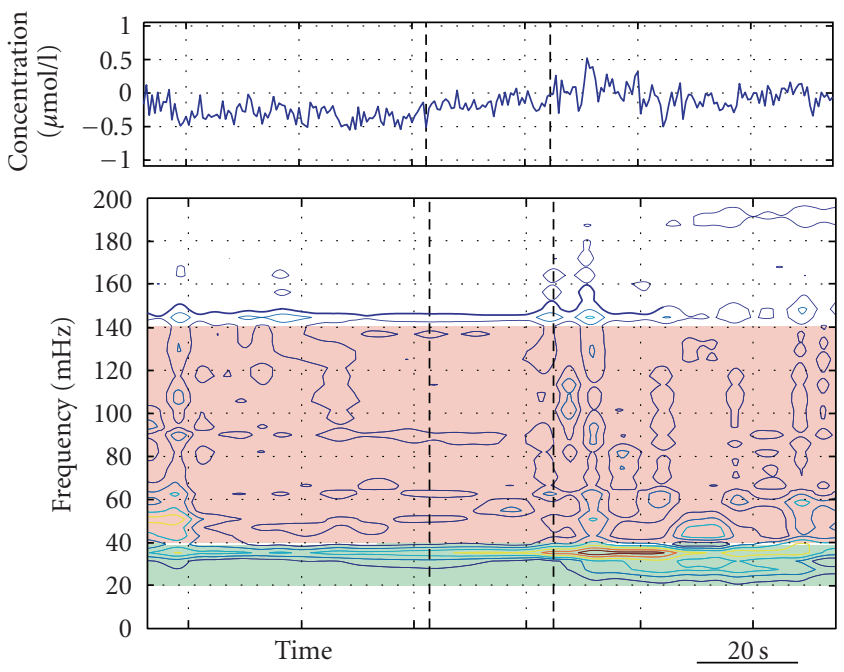

(b)

FIgURE 5: Time-frequency representations of $\mathrm{O}_{2} \mathrm{Hb}$ (a) and $\mathrm{HHb}$ (b) concentration signals during $\mathrm{BH}$. The graphs are relative to a subject suffering from migraine with aura. The vertical dashed lines mark the $\mathrm{BH}$ onset and offset. The red rectangle indicates the LF band $(40-140 \mathrm{mHz})$, the green the VLF band $(20-40 \mathrm{mHz})$. It is possible to notice that $\mathrm{BH}$ seems to neatly increase the LF band power of the signals.

distribution $D_{x x}(t, f)$ belonging to the Cohen's class can be given as

$$
\begin{aligned}
D_{x x}(t, f)=\iiint_{-\infty}^{+\infty} x & \left(t^{\prime}-\frac{\tau}{2}\right) x^{*}\left(t^{\prime}+\frac{\tau}{2}\right) \\
& \times g(\tau, \theta) e^{-j 2 \pi \theta\left(t^{\prime}-t\right)} e^{-j 2 \pi f \tau} d t^{\prime} d \theta d \tau,
\end{aligned}
$$

where $x(t)$ is the signal under analysis, $\theta$ and $\tau$ are the frequency and time lags, respectively, and $g(\tau, \theta)$ is the kernel of the time-frequency distribution. We used the Choi-Williams distribution (CW) [27], whose kernel is expressed as $g(\tau, \theta)=e^{-\left(\tau^{2} \theta^{2} / \sigma\right)}$, where $\sigma$ is a selectivity
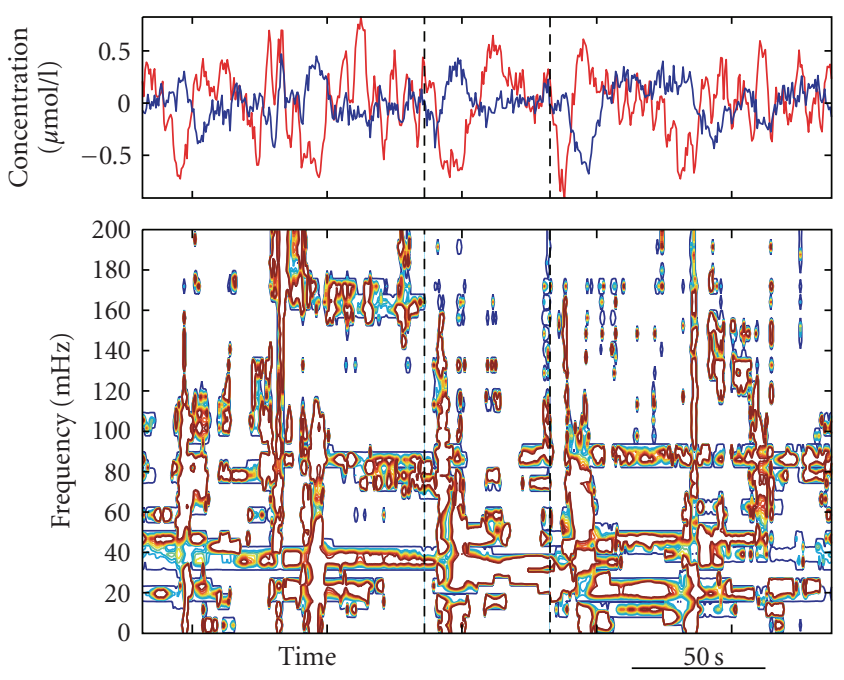

FIgure 6: Time-frequency Squared Coherence Function (SCF) between the $\mathrm{O}_{2} \mathrm{Hb}$ (red line) and $\mathrm{HHb}$ (blue line) concentration signals during $\mathrm{BH}$. The graph is relative to a subject suffering from migraine with aura. The vertical dashed lines mark the $\mathrm{BH}$ onset and offset. The SCF is represented by a contour plot. The white spots indicate time instants and frequency values where the coherence between the signals approximates 1 .

parameter. Large values of $\sigma$ determine a lower attenuation of the interference terms, whereas small values make the representation cleaner. However, small $\sigma$ values might result in an evident loss of spectral resolution in the time-frequency plane. We used the CW transform since it proved effective in the analysis of biological signals and was used in a pilot previous study on NIRS signals [28].

We computed the signals time-frequency distributions by means of a custom developed toolbox running in Matlab (TheMathworks, Natick, MA, USA) environment. This toolbox first computes the instantaneous autocorrelation function of a time series $x[n]$, then computes the corresponding ambiguity function by an inverse Fourier transform, applies the CW kernel, and finally computes the $D_{x x}(t, f)$ by a double Fourier transform from the lags to the time and frequency variables. Our algorithm discretizes the instantaneous autocorrelation function $R_{x x}(t, \tau)=x(t-$ $\tau / 2) x^{*}(t+\tau / 2)$ defined by (2) according to the following formula:

$$
R_{x x}[n, k]=x[n-k] x^{*}[n+k],
$$

where $n$ represents the discrete time and $k$ the time lag. The definition in (3) is symmetrical with respect to the time lag, but it is clearly subjected to possible frequency aliasing. In fact, the symmetrical definition of the correlation product determines a subsampling of the $\tau$ axis of a factor equal to 2 . Therefore, the maximum frequency that can be represented by this definition is equal to $f_{s} / 4$, being $f_{s}$ the sampling frequency. Since the bandwidth upper limit of our NIRS signals was equal to about $200 \mathrm{mHz}$, being $2 \mathrm{~Hz}$ the sampling rate, our time-frequency representations did not suffer from aliasing. 
We also computed the time-frequency Squared Coherence Function (SCF) between the $\mathrm{O}_{2} \mathrm{Hb}$ and the $\mathrm{HHb}$ concentration signals. Being $x(t)$ the $\mathrm{O}_{2} \mathrm{Hb}$ concentration signal and $y(t)$ the $\mathrm{HHb}$, the SCF between the two signals was defined as

$$
\operatorname{SCF}_{x y}(t, f)=\frac{\left|D_{x y}(t, f)\right|^{2}}{D_{x x}(t, f) \cdot D_{y y}(t, f)},
$$

where $D_{x y}(t, f)$ is the cross time-frequency CW representation of the $\mathrm{O}_{2} \mathrm{Hb}$ and $\mathrm{HHb}$ concentration signals, $D_{x x}(t, f)$ is the time-frequency $\mathrm{CW}$ representation of the $\mathrm{O}_{2} \mathrm{Hb}$ signal, and $D_{y y}(t, f)$ that of the $\mathrm{HHb}$ signal.

All the auto and cross time-frequency distributions were computed on a 256 seconds time window, with the event (either BH of HYP) centered in the middle of the window (see Figure 1), so that the theoretical spectral resolution was better than $4 \mathrm{mHz}$. This value was a good compromise between the need for a suitable separation of the VLF and LF bands and for keeping the experimental protocol sufficiently short.

All the signals were converted to their analytical representation with zero mean and no trend. Trends were removed by using high-pass filtering (Chebychev filter, with ripple in the stopband and cutoff frequency equal to $15 \mathrm{mHz}$ ).

Figure 2 reports an example of time-frequency representation (depicted by means of a 6-levels contour plot) of the $\mathrm{HHb}$ signal of a healthy woman performing $\mathrm{BH}$. The upper panel shows the time course of the $\mathrm{HHb}$ concentration signal, the lower the CW representation ( $\sigma$ was kept equal to 0.5 for all the signals). The vertical dashed lines represent the $\mathrm{BH}$ onset and offset. The green rectangle overlaid to the image shows the VLF frequency band, the red rectangle the LF. In this specific subject, there is a noticeable increase in the power of the LF band after $\mathrm{BH}$.

2.3. Subjects. We tested 5 healthy women taken as controls (age: $30.2 \pm 12.1$ years), 14 women suffering from MwoA (age: $44.4 \pm 9.7$ years) and 49 women suffering from MwA (age: $38.0 \pm 12.1$ years), for a total of 68 subjects. Migraine with and without aura was diagnosed according to the criteria of the International Headache Society [29]. Migraine subjects were tested in the interictal period, when they were free of pain.

The study received the approval from the Review Institutional Committee of the Gradenigo Hospital of Torino (Italy), where all the experiments were conducted. All the subjects were instructed about the purposes of the study and signed an informed consent prior of being tested.

2.4. Statistical Analysis. We organized the data in a matrix containing the 68 subjects as rows and 26 measured variables as columns. On each subject, we measured the following variables derived from the time-frequency representations:

(i) the $\mathrm{HHb}$ and $\mathrm{O}_{2} \mathrm{Hb}$ power in the VLF and LF bands $\left(\mathrm{P}_{\mathrm{VLF}}\right.$ and $\mathrm{P}_{\mathrm{LF}}$ ), before and after $\mathrm{BH}$ (for a total of 8 variables) averaged on a 60 seconds window expressed in percentage with respect to the total signal power;

(ii) the $\mathrm{HHb}$ and $\mathrm{O}_{2} \mathrm{Hb}$ power in the VLF and LF bands $\left(\mathrm{P}_{\mathrm{VLF}}\right.$ and $\mathrm{P}_{\mathrm{LF}}$ ), before and after HYP (for a total of 8 variables) averaged on a 60 seconds window expressed in percentage with respect to the total signal power;

(iii) the $\mathrm{O}_{2} \mathrm{Hb}$ and $\mathrm{HHb} \mathrm{SCF}$ value in the two bands $\left(\mathrm{SCF}_{\mathrm{VLF}}\right.$ and $\left.\mathrm{SCF}_{\mathrm{LF}}\right)$, before and after $\mathrm{BH}$ and $\mathrm{HYP}$ (for a total of 8 variables) averaged on a 60 seconds window;

(iv) the $\mathrm{BHI}$ index for $\mathrm{HHb}$ and $\mathrm{O}_{2} \mathrm{Hb}$ signals (2 variables). These measures are standard in the cerebral assessment and derive from the concentration signals time course. Considering the $\mathrm{O}_{2} \mathrm{Hb}$ signal, the $\mathrm{BHI}_{\mathrm{O} 2 \mathrm{Hb}}$ is defined as the percent variation of the $\mathrm{O}_{2} \mathrm{Hb}$ concentration as effect of $\mathrm{BH}$, normalized with respect to the $\mathrm{BH}$ duration $[14,30]$.

The first column of Table 1 summarizes the measured variables. The signal power in the VLF and LF bands was computed by integration of the corresponding timefrequency representation.

We used ANOVA analysis to extract the most significant variables from the set of parameters of Table 1 (first column) that explained the data distribution based on pathology.

We performed a one-way ANOVA analysis considering the pathology as independent variable and the remaining values as dependent variables, one at a time. Among the variables, we removed all the observations with $P$ value greater than $10 \%$. This allowed for a reduction of the number of variables and for avoiding an overfitting of the system with strongly correlated variables. Then, we performed an unsupervised analysis on the remaining variables to represent our sample population on the basis of the measured parameters.

Specifically, we performed a principal component analysis (PCA) in order to better represent the data information in a transformed domain with lower dimensionality. PCA generates a set of new variables, called principal components (PCs), as linear combination of the original ones. PCA was used to observe which spectral parameters could be of help in clustering the subjects of our mixed sample population.

\section{Results}

Table 1 reports the results of the ANOVA analysis considering as the subject pathology independent variable and the 26 previously described measurements as dependent variables. We chose to keep only the variables resulting in a $P$ value lower than $10 \%$ (such variables are marked by an asterisk in the second column of Table 1). The ANOVA analysis restituted five variables: the $\mathrm{O}_{2} \mathrm{Hb}$ power in the LF band after $\mathrm{BH}\left(\mathrm{P}_{\mathrm{LF}}\right.$ postBH $\left.-\mathrm{O}_{2} \mathrm{Hb}\right)$, the $\mathrm{HHb}$ power in the LF band after $\mathrm{BH}\left(\mathrm{P}_{\mathrm{LF}}\right.$ postBH $\left.-\mathrm{HHb}\right)$, the $\mathrm{O}_{2} \mathrm{Hb}$ power in the LF band before HYP $\left(\mathrm{P}_{\mathrm{LF}}\right.$ preHYP $\left.-\mathrm{O}_{2} \mathrm{Hb}\right)$, the coherence value in the VLF band before HYP ( $\mathrm{SCF}_{\mathrm{VLF}}$ preHYP), and the $\mathrm{BHI}_{\mathrm{HHb}}$. These variables are the ones that best describe 
TABLE 1: ANOVA results. Results of one-way ANOVA analysis performed considering as independent variable the subject pathology (no migraine, MwA, or MwoA). The first column reports the dependent variables and the second column reports the associated $P$-value. The significant results $(P<10 \%)$ are indicated with asterisk.

\begin{tabular}{ll}
\hline Dependent variable & $P$ value \\
\hline $\mathrm{P}_{\mathrm{VLF}}$ preBH $-\mathrm{O}_{2} \mathrm{Hb}$ & $58.63 \%$ \\
$\mathrm{P}_{\mathrm{VLF}}$ postBH $-\mathrm{O}_{2} \mathrm{Hb}$ & $21.05 \%$ \\
$\mathrm{P}_{\mathrm{LF}}$ preBH $-\mathrm{O}_{2} \mathrm{Hb}$ & $69.43 \%$ \\
$\mathrm{P}_{\mathrm{LF}}$ postBH $-\mathrm{O}_{2} \mathrm{Hb}$ & $8.92 \% *$ \\
$\mathrm{P}_{\mathrm{VLF}}$ preBH $-\mathrm{HHb}$ & $86.72 \%$ \\
$\mathrm{P}_{\mathrm{VLF}}$ postBH $-\mathrm{HHb}$ & $56.04 \%$ \\
$\mathrm{P}_{\mathrm{LF}}$ preBH $-\mathrm{HHb}$ & $99.15 \%$ \\
\hline $\mathrm{P}_{\mathrm{LF}}$ postBH $-\mathrm{HHb}$ & $5.32 \% *$ \\
$\mathrm{P}_{\mathrm{VLF}}$ preHYP $-\mathrm{O}_{2} \mathrm{Hb}$ & $11.91 \%$ \\
$\mathrm{P}_{\mathrm{VLF}}$ postHYP $-\mathrm{O}_{2} \mathrm{Hb}$ & $78.48 \%$ \\
$\mathrm{P}_{\mathrm{LF}}$ preHYP $-\mathrm{O}_{2} \mathrm{Hb}$ & $3.22 \% *$ \\
$\mathrm{P}_{\mathrm{LF}}$ postHYP $-\mathrm{O}_{2} \mathrm{Hb}$ & $90.51 \%$ \\
$\mathrm{P}_{\mathrm{VLF}}$ preHYP $-\mathrm{HHb}$ & $87.42 \%$ \\
$\mathrm{P}_{\mathrm{VLF}}$ postHYP $-\mathrm{HHb}$ & $90.66 \%$ \\
$\mathrm{P}_{\mathrm{LF}}$ preHYP $-\mathrm{HHb}$ & $14.03 \%$ \\
$\mathrm{P}_{\mathrm{LF}}$ post $\mathrm{HYP}-\mathrm{HHb}$ & $61.34 \%$ \\
\hline $\mathrm{S}_{\mathrm{VLF}}$ preBH & $86.72 \%$ \\
$\mathrm{~S}_{\mathrm{VLF}}$ postBH & $85.30 \%$ \\
$\mathrm{~S}_{\mathrm{LF}}$ preBH & $97.94 \%$ \\
$\mathrm{~S}_{\mathrm{LF}}$ postBH & $14.47 \%$ \\
$\mathrm{~S}_{\mathrm{VLF}}$ preHYP & $0.49 \% *$ \\
$\mathrm{~S}_{\mathrm{VLF}}$ post $\mathrm{HYP}$ & $57.99 \%$ \\
$\mathrm{~S}_{\mathrm{LF}}$ preHYP & $35.04 \%$ \\
$\mathrm{~S}_{\mathrm{LF}}$ postHYP & $20.15 \%$ \\
\hline $\mathrm{BHI}_{\mathrm{O} 2}$ & $50.04 \%$ \\
$\mathrm{BHI}$ & $0.02 \% *$ \\
\hline
\end{tabular}

TABLE 2: PCA components. Weights of the three principal components of the PCA analysis in function of the five original variables.

\begin{tabular}{lccc}
\hline Variable & Component 1 & Component 2 & Component 3 \\
\hline $\mathrm{P}_{\mathrm{LF}}$ postBH - $\mathrm{O}_{2} \mathrm{Hb}$ & -0.61 & -0.08 & -0.21 \\
$\mathrm{P}_{\mathrm{LF}}$ postBH - HHb & -0.61 & 0.21 & -0.11 \\
$\mathrm{P}_{\mathrm{LF}}$ preHYP - $\mathrm{O}_{2} \mathrm{Hb}$ & -0.47 & -0.17 & 0.027 \\
$\mathrm{~S}_{\mathrm{VLF}}$ preHYP & 0.17 & 0.36 & -0.89 \\
$\mathrm{BHI}_{\mathrm{HHb}}$ & -0.08 & 0.89 & 0.38 \\
\hline
\end{tabular}

the sample population and, therefore, are expected to be significantly different in the three subgroups of subjects.

PCA was conducted on a data set consisting of a matrix with 68 rows (patients) and the above-mentioned 5 observations. All the variables were standardized by removing their mean value and by normalizing with respect to their standard deviation. We chose to represent the data using the first 3 PCs that explained $80.7 \%$ of the total variance of the data. Table 2 reports the weights of the five variables for the three components. The first component is dominated by the $\mathrm{O}_{2} \mathrm{Hb}$ and $\mathrm{HHb} \mathrm{LF}$ power after $\mathrm{BH}$, the second by the $\mathrm{BHI}_{\mathrm{HHb}}$, and the third by the coherence value in the VLF band before HYP.

Figure 3 reports the distribution of the original data set on the hyperplanes formed by component 1 and 2 (upper graph), and component 1 and 3 (lower graph). Green circles represent the MwoA subjects, yellow circles the MwA, and the red squares the healthy subjects (i.e., the controls). The continuous blue lines represent the projection of the original variables on the hyperplanes. The graphs of Figure 3 are mixed representations: the circles and squares represent the subjects in the new systems originated by the PCs (i.e., it is a scores/scores plot), whereas the blue lines with the text labels represent the original variable in function of the new coordinate systems (i.e., it is a loading/loading plot). It can be observed that MwoA subjects (green circles) are clustered relatively far from the MwA and healthy subjects. With reference to Figure 3 upper panel, the most characterizing original variables for MwoA subjects are those directed towards right (i.e., the positive axis of Component 1): $\mathrm{P}_{\mathrm{LF}}$ postBH $-\mathrm{O}_{2} \mathrm{Hb}, \mathrm{P}_{\mathrm{LF}}$ postBH $-\mathrm{HHb}$, and $\mathrm{P}_{\mathrm{LF}}$ preHYP - $\mathrm{O}_{2} \mathrm{Hb}$. Specifically, MwoA subjects should have lower values of the above-mentioned three variables with respect to the other subjects of the sample population, since in the loading/loading plot the blue lines mark the increasing direction of the original variables in the PCs space.

Figure 4 depicts the CW time-frequency representation of the $\mathrm{O}_{2} \mathrm{Hb}$ (Figure 4(a)) and $\mathrm{HHb}$ (Figure 4(b)) concentration signals of a MwoA performing $\mathrm{BH}$. The vertical dashed lines mark the onset and offset of the $\mathrm{BH}$. The overlaid red rectangle indicates the LF band on the timefrequency plane, the green the VLF. Considering the timefrequency representation after $\mathrm{BH}$, it is possible to notice that there is a low signal power in the red rectangle both in the $\mathrm{O}_{2} \mathrm{Hb}$ and $\mathrm{HHb}$ graphs. Figure 5 depicts the CW timefrequency representation of the $\mathrm{BH}$ performed by a MwA subject, with analogous coding of Figure 4. In Figures 5(a) and $5(\mathrm{~b})$, it is evident that after $\mathrm{BH}$ the power content of the $\mathrm{O}_{2} \mathrm{Hb}$ and $\mathrm{HHb}$ is higher than for the MwoA subject. Particularly, in Figure 5(b) it can be noticed that the $\mathrm{HHb}$ concentration signal after $\mathrm{BH}$ shows diffuse components up to $100-140 \mathrm{mHz}$. BH enforces the LF oscillations in the MwA subject, whereas it depresses the LF content in the MwoA subject.

\section{Discussion}

The time-frequency analysis of NIRS signals recorded during active maneuvers allowed for the unsupervised analysis of a mixed population consisting of healthy women, women suffering from MwA, and women suffering from MwoA. To the best of our knowledge, this is the first study coupling time-frequency analysis applied to the NIRS signals and a multivariate analysis for the characterization of a neurological disorder.

In a previous study, we showed that women suffering from MwA revealed an impaired carbon dioxide regulatory mechanism with respect to controls [28]. Specifically, we found that BH caused an increase in the LF band power on the $\mathrm{HHb}$ signal that was statistically lower than the 
increase of controls. This result was obtained by means of the CW transform applied to the NIRS signals recorded on a 256 seconds time window in which the subject performed $\mathrm{BH}$. In this study, we enlarged the recording window and adopted a longer testing protocol that incorporates the HYP too. However, despite the enlargement of the test, our previous results are confirmed. Figure 3 shows that MwA subjects are located in a hyperplane region corresponding to lower values of $\mathrm{HHB}$ power in the LF band after $\mathrm{BH}$ than controls. Even though such difference is not neat, a significant number of MwA subjects shows a behavior similar to that we documented in our previous study [28].

The novel result of this study relies in the observation that MwoA sufferers seem characterized by a completely different oxygenation pattern. After $\mathrm{BH}$, they had a very low power in the LF band (Figure 4) both on the $\mathrm{O}_{2} \mathrm{Hb}$ and $\mathrm{HHb}$ concentration signals. A statistical test conducted on the MwoA subsample revealed that $\mathrm{BH}$ did not increase the LF power in the NIRS signals (Student's t-test, $P<.01$ ). The other variables discriminating the MwoA patients from the rest of the population were the $\mathrm{BH}_{\mathrm{HHb}}$, the power in the $\mathrm{LF}$ band of the $\mathrm{O}_{2} \mathrm{Hb}$ signal before HYP, and the coeherence value between $\mathrm{O}_{2} \mathrm{Hb}$ and $\mathrm{HHb}$ in the VLF band before HYP. Except the $\mathrm{BHI}_{\mathrm{HHb}}$, which is derived from the time course of the signals, the other discriminant variables are linked to the frequency content of the NIRS oxygenation signals.

From a methodological point of view, the use of timefrequency analysis proved essential in the characterization of the subjects' cerebral hemodynamics during active maneuvers. Active tests such as $\mathrm{BH}$ and HYP are needed to test the regulatory mechanisms. However, they introduce evident nonstationarities in the recorded signals. As previously observed [21], cerebral autoregulation is based on two distinct mechanisms, which originate the VLF and LF bands. During the regulatory action, the power in these bands changes, thus making the NIRS signals strongly nonstationary. Since the VLF and LF bands are very close and centered at very low frequency values (ranging from about $20 \mathrm{mHz}$ to $140 \mathrm{mHz}$ ), a frequency analysis tool with high spectral resolution is required.

The bilinear time-frequency distributions belonging to the Cohen's class are a good choice, since they couple a good and constant resolution on the frequency axis to the effective possibility of interference terms rejection. We analyzed our signals on a 265 seconds time window incorporating the active stimulus (either BH or HYP). Obrig et al. in 2000 studied the spontaneous oscillations detected by NIRS during apnea and visual stimulation by using a 102.4 seconds time window. They used the Welch periodogram with 512 Hanning-type window and 128 points of overlap [21]. Therefore, having a sampling rate of $10 \mathrm{~Hz}$, their spectral resolution was slightly better than $20 \mathrm{mHz}$. They had to limit their spectral resolution due to the nonstationary nature of NIRS signals: they recorded signals epochs before and after the stimulations, with the hypothesis that, in such epochs, the signals could be considered at least as wide-sense stationary processes. The use of time-frequency distributions does not limit the resolution that can be acquired and does not require any hypothesis on the nature of the NIRS signals.
We processed our data by using a 102.4 seconds time window, but we found that the spectral resolution was too poor to distinguish the VLF from the LF band. Therefore, the PCA analysis was insensible to pathology and resulted in a mixed representation.

One of the encountered problems is represented by the slow trends the concentrations signals show during time. Often, in correspondence of the stimulus, relatively big and fast (see Figure 1(a)) or slow (see Figure 1(b)) trends can be observed on the signals. Such trends might mask the VLF band and make the frequency analysis little reliable. We found a great variability of such trends among subjects. We tried three different detrending techniques: (i) the 3rd order polynomial detrending, (ii) the smoothness priors method proposed by Tarvainen et al. [31], and (iii) the traditional high-pass filtering. We found that polynomial detrending was not suitable to our data, since for trends generated by HYP it was sufficient an order equal to 3, but for the abrupt trends of caused by the end of the $\mathrm{BH}$, an order of 5 or more was required. The smoothness priors method was developed for heart rate variability analysis [31] and it implements an automated high-pass-like filter. However, the resulting filter possessed a too high cutoff frequency that attenuated almost completely the VLF band. Therefore, we used a Chebychev type ARMA filter and kept the detrending strategy equal for all the subjects and all the events.

We computed the time-frequency SCF between the $\mathrm{O}_{2} \mathrm{Hb}$ and $\mathrm{HHb}$ signals. Figure 6 reports an example of SCF computed during the $\mathrm{BH}$ of a MwA patient. The SCF is represented by level curves; the white spots mark the time instants and the frequency values for which there is coherence between the $\mathrm{O}_{2} \mathrm{Hb}$ and $\mathrm{HHb}$ signals. In our study, the SCF value was never significant after a stimulus, but only before HYP and only in the VLF band. The coherence value of the VLF band before HYP dominates the third component of the PCA (see Figure 3 lower panel and Table 2). This coherence value is slightly higher in MwA than in MwoA and control subjects (Figure 3, lower panel). Further work is required in order to validate the importance of the coherence in pathologic versus healthy NIRS recordings. However, this paper is the first attempt of bringing the timefrequency analysis of coherence in NIRS signals into a clinical evaluation protocol.

Finally, the computational cost of our time-frequency implementation is of about 5 seconds for a 512 points signal epoch (Matlab 7.04 running on a $2.5 \mathrm{GHz}$ dual-G5 Apple PowerPc equipped by 8 GB of RAM). Therefore, considered that the experimental protocols lasts slightly less than 10 minutes, our analysis procedure can be carried out in realtime. The herein proposed time-frequency methodology is currently under testing in the Department of Neuroscience of the Gradenigo Hospital of Torino, Italy.

\section{Conclusion}

The time-frequency-based analysis of NIRS signals during active maneuvers allowed for the high-resolution quantification of the signals power in the VLF and LF bands. Such values demonstrated a neat difference in the cerebral 
hemodynamics of migraine sufferers with and without aura. Particularly, MwoA sufferers are characterized by low power of the LF band when performing breath-holding, whereas MwA subjects shows higher values.

Our study showed that the time-frequency analysis of these signals is crucial if the assessment of cerebral hemodynamics is the clinical issue. In fact, traditional spectral analysis makes such assessment impossible due scarce spectral resolution coupled to the effects of signals' nonstationarity.

This time-frequency-based methodology is a first attempt of bringing the spectral analysis of NIRS signals into a clinical application and it is currently under validation. We are now improving our methodology by considering possible system nonlinearities and higher-order statistics analysis tools.

\section{Abbreviations}

NIRS: Near-InfraRed Spectroscopy

BH: Breath-Holding

HYP: HYPerventilation

$\mathrm{O}_{2} \mathrm{Hb}$ : Oxygenated hemoglobin

HHb: Reduced (deoxygenated) Hemoglobin

CW: Choi-Williams time-frequency transform

SCF: Squared Coherence Function

LF: Low Frequency oscillations

VLF: Very Low Frequency oscillations

MwA: Migraine with Aura

MwoA: Mirgaine without Aura.

\section{Acknowledgment}

The authors would like to thank Dr. Gianfranco Grippi (Department of Neuroscience, Gradenigo Hospital, Torino, Italy) for the support in the transcranial Doppler assessment of the subjects.

\section{References}

[1] R. B. Panerai, "Cerebral autoregulation: from models to clinical applications," Cardiovascular Engineering, vol. 8, no. 1, pp. 42-59, 2008.

[2] M. Daffertshofer and M. Hennerici, "Cerebrovascular regulation and vasoneuronal coupling," Journal of Clinical Ultrasound, vol. 23, no. 2, pp. 125-138, 1995.

[3] W. Rudziński, M. Swiat, M. Tomaszewski, and J. Krejza, "Cerebral hemodynamics and investigations of cerebral blood flow regulation," Nuclear Medicine Review, vol. 10, no. 1, pp. 29-42, 2007.

[4] R. Aaslid, "Cerebral autoregulation and vasomotor reactivity," Frontiers of Neurology and Neuroscience, vol. 21, pp. 216-228, 2006.

[5] P. N. Ainslie and J. Duffin, "Integration of cerebrovascular $\mathrm{CO}_{2}$ reactivity and chemoreflex control of breathing: mechanisms of regulation, measurement, and interpretation," American Journal of Physiology, vol. 296, no. 5, pp. R1473R1495, 2009.

[6] V. C. Urrutia and R. J. Wityk, "Blood pressure management in acute stroke," Neurologic Clinics, vol. 26, no. 2, pp. 565-583, 2008.
[7] R. Aaslid, T. M. Markwalder, and H. Nornes, "Noninvasive transcranial Doppler ultrasound recording of flow velocity in basal cerebral arteries," Journal of Neurosurgery, vol. 57, no. 6, pp. 769-774, 1982.

[8] W. Liboni, G. Allais, O. Mana et al., "Transcranial Doppler for monitoring the cerebral blood flow dynamics: normal ranges in the Italian female population," Panminerva Medica, vol. 48, no. 3, pp. 187-191, 2006.

[9] D. A. Benaron, S. R. Hintz, A. Villringer et al., "Noninvasive functional imaging of human brain using light," Journal of Cerebral Blood Flow and Metabolism, vol. 20, no. 3, pp. 469477, 2000.

[10] M. Firbank, E. Okada, and D. T. Delpy, "Investigation of the effect of discrete absorbers upon the measurement of blood volume with near-infrared spectroscopy," Physics in Medicine and Biology, vol. 42, no. 3, pp. 465-477, 1997.

[11] A. Kastrup, T.-Q. Li, G. H. Glover, and M. E. Moseley, "Cerebral blood flow-related signal changes during breathholding," American Journal of Neuroradiology, vol. 20, no. 7, pp. 1233-1238, 1999.

[12] W. Liboni, F. Molinari, G. Allais et al., "Why do we need NIRS in migraine?" Neurological Sciences, vol. 28, no. 2, pp. S222S224, 2007.

[13] H. Obrig, R. Wenzel, M. Kohl et al., "Near-infrared spectroscopy: does it function in functional activation studies of the adult brain?" International Journal of Psychophysiology, vol. 35, no. 2-3, pp. 125-142, 2000.

[14] F. Molinari, W. Liboni, G. Grippi, and E. Negri, "Relationship between oxygen supply and cerebral blood flow assessed by transcranial Doppler and near-infrared spectroscopy in healthy subjects during breath-holding," Journal of NeuroEngineering and Rehabilitation, vol. 3, article no. 16, 2006.

[15] A. Piepgras, P. Schmiedek, G. Leinsinger, R. L. Haberl, C. M. Kirsch, and K. M. Einhaupl, "A simple test to assess cerebrovascular reserve capacity using transcranial Doppler sonography and acetazolamide," Stroke, vol. 21, no. 9, pp. 1306-1311, 1990.

[16] E. B. Ringelstein, S. Van Eyck, and I. Mertens, "Evaluation of cerebral vasomotor reactivity by various vasodilating stimuli: comparison of $\mathrm{CO}_{2}$ to acetazolamide," Journal of Cerebral Blood Flow and Metabolism, vol. 12, no. 1, pp. 162-168, 1992.

[17] M. S. V. Elkind and A. I. Scher, "Migraine and cognitive function: some reassuring news," Neurology, vol. 64, no. 4, pp. 590-591, 2005.

[18] M. C. Kruit, M. A. van Buchem, P. A. M. Hofman et al., "Migraine as a risk factor for subclinical brain lesions," Journal of the American Medical Association, vol. 291, no. 4, pp. 427434, 2004.

[19] G. E. Tietjen, "Migraine as a systemic vasculopathy," Cephalalgia, vol. 29, no. 9, pp. 989-996, 2009.

[20] F. Vernieri, F. Tibuzzi, P. Pasqualetti et al., "Increased cerebral vasomotor reactivity in migraine with aura: an autoregulation disorder? A transcranial Doppler and near-infrared spectroscopy study," Cephalalgia, vol. 28, no. 7, pp. 689-695, 2008.

[21] H. Obrig, M. Neufang, R. Wenzel et al., "Spontaneous low frequency oscillations of cerebral hemodynamics and metabolism in human adults," NeuroImage, vol. 12, no. 6, pp. 623-639, 2000.

[22] U. Sliwka, S. Harscher, R. R. Diehl, R. van Schayck, W. D. Niesen, and C. Weiller, "Spontaneous oscillations in cerebral blood flow velocity give evidence of different autonomic dysfunctions in various types of headache," Headache, vol. 41, no. 2, pp. 157-163, 2001. 
[23] A. Duncan, J. H. Meek, M. Clemence et al., "Optical pathlength measurements on adult head, calf and forearm and the head of the newborn infant using phase resolved optical spectroscopy," Physics in Medicine and Biology, vol. 40, no. 2, pp. 295-304, 1995.

[24] T. S. Leung, I. Tachtsidis, M. Smith, D. T. Delpy, and C. E. Elwell, "Measurement of the absolute optical properties and cerebral blood volume of the adult human head with hybrid differential and spatially resolved spectroscopy," Physics in Medicine and Biology, vol. 51, no. 3, pp. 703-717, 2006.

[25] E. Okada, M. Firbank, M. Schweiger, S. R. Arridge, M. Cope, and D. T. Delpy, "Theoretical and experimental investigation of near-infrared light propagation in a model of the adult head," Applied Optics, vol. 36, no. 1, pp. 21-31, 1997.

[26] L. Cohen, Time-Frequency Analysis, Prentice-Hall, New York, NY, USA, 1995.

[27] H. Choi and W. J. Williams, "Improved time-frequency representation of multicomponent signals using exponential kernels," IEEE Transactions on Acoustics, Speech, and Signal Processing, vol. 37, no. 6, pp. 862-871, 1989.

[28] W. Liboni, F. Molinari, G. Allais et al., "Spectral changes of near-infrared spectroscopy signals in migraineurs with aura reveal an impaired carbon dioxide-regulatory mechanism," Neurological Sciences, vol. 30, no. 1, pp. S105-S107, 2009.

[29] "Classification and diagnostic criteria for headache disorders, cranial neuralgias and facial pain. Headache Classification Committee of the International Headache Society," Cephalalgia, vol. 8, supplement 7, pp. 1-96, 1988.

[30] F. Vernieri, F. Tibuzzi, P. Pasqualetti et al., "Transcranial Doppler and near-infrared spectroscopy can evaluate the hemodynamic effect of carotid artery occlusion," Stroke, vol. 35, no. 1, pp. 64-70, 2004.

[31] M. P. Tarvainen, P. O. Ranta-aho, and P. A. Karjalainen, "An advanced detrending method with application to HRV analysis," IEEE Transactions on Biomedical Engineering, vol. 49, no. 2, pp. 172-175, 2002. 